\title{
Bacterial analogs to cholesterol affect dimerization of proteorhodopsin and modulates preferred dimer interface
}

\author{
Eric Sefah and Blake Mertz
}

Corresponding author: blake.mertz@mail.wvu.edu

This PDF file includes:

Figs. S1 to S6

Tables S1 to S4 

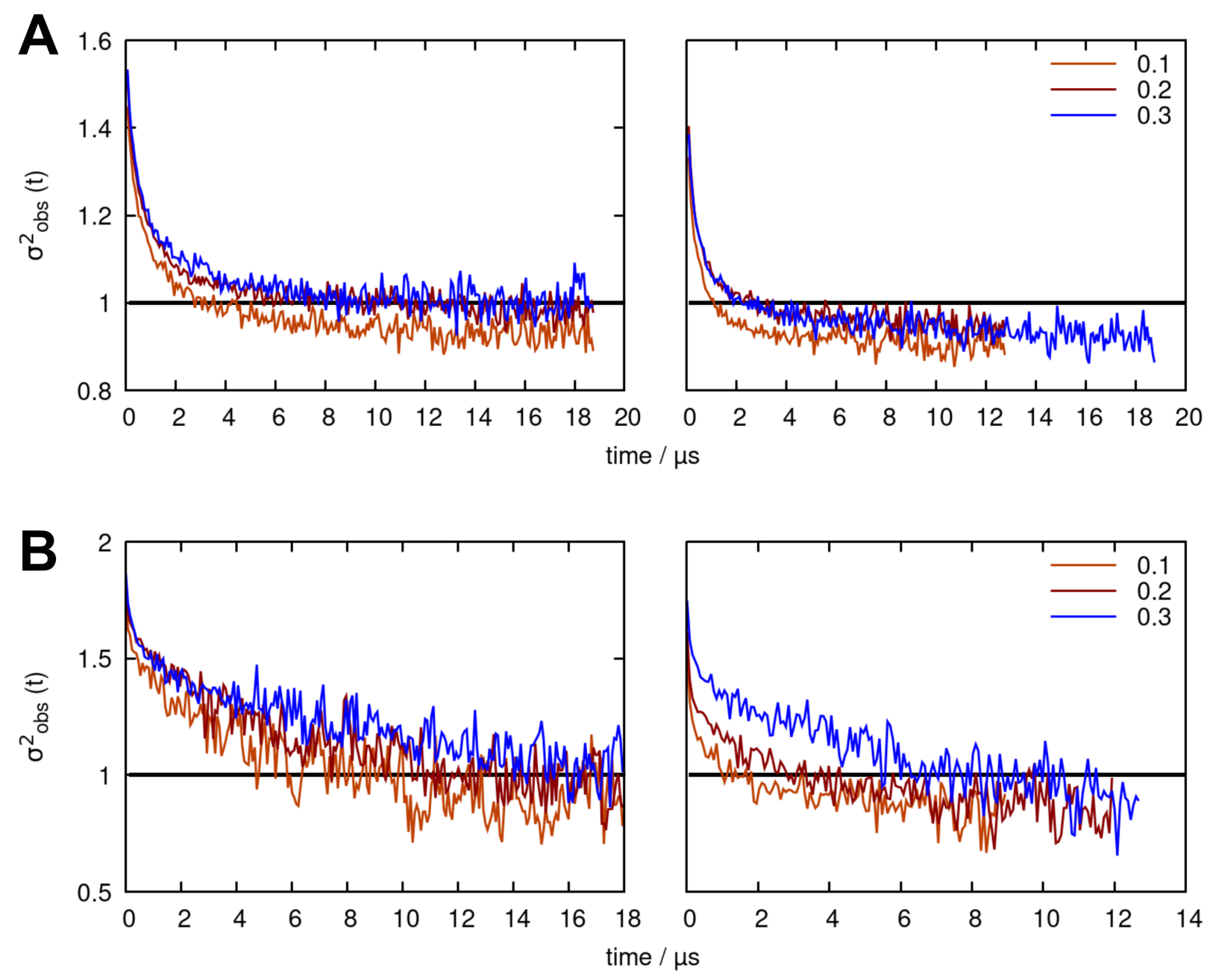

Figure S1: Decorrelation times for hopanoid systems show that equilibrium occurs in under ten microseconds. A) Decorrelation times for BHT (left) and DPT (right) in a monomeric PR system as a function of concentration of each respective hopanoid. B) Decorrelation times for BHT (left) and DPT (right) in a dimeric PR system as a function of concentration of each respective hopanoid. 


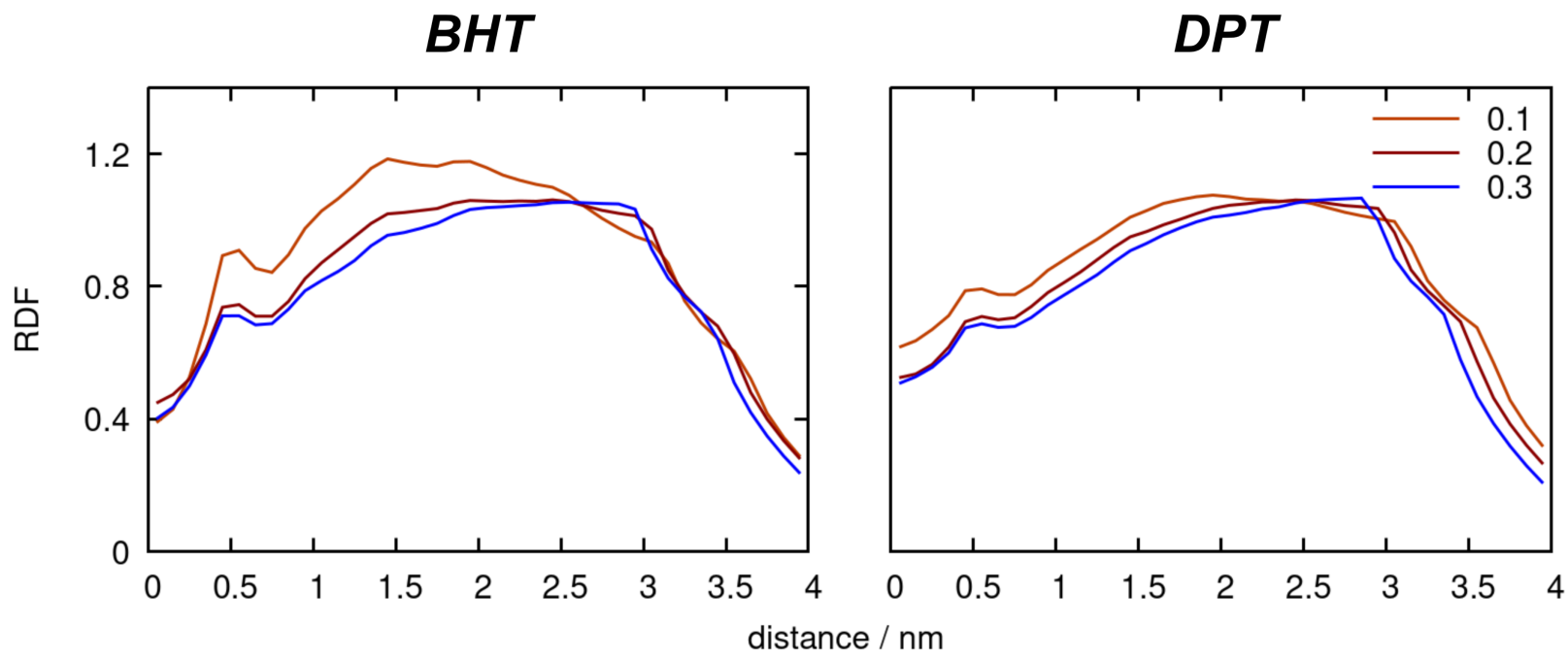

Figure S2: First shell of hopanoids around PR is within $1 \mathbf{n m}$ of the protein. Radial distribution function (RDF) for BHT (left) and DPT (right) in a monomeric system as a function of concentration of each respective hopanoid.

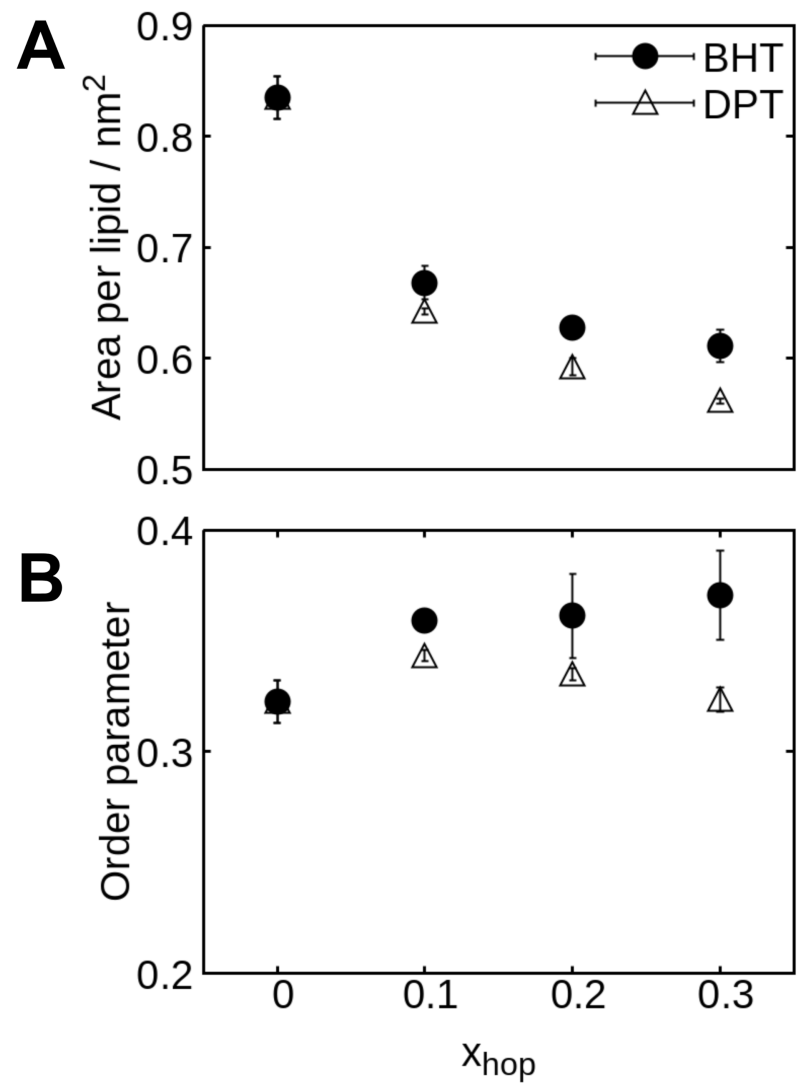

Figure S3: Trends in 1-palmitoyl-2-oleoyl-sn-3-glycero-phosphoglycerol (POPG) for addition of BHT or DPT to proteorhodopsin (PR) systems. A) Area per lipid of POPG as a function of mol \% of hopanoid. B) Order parameter of POPG as a function of mol \% of hopanoid. 


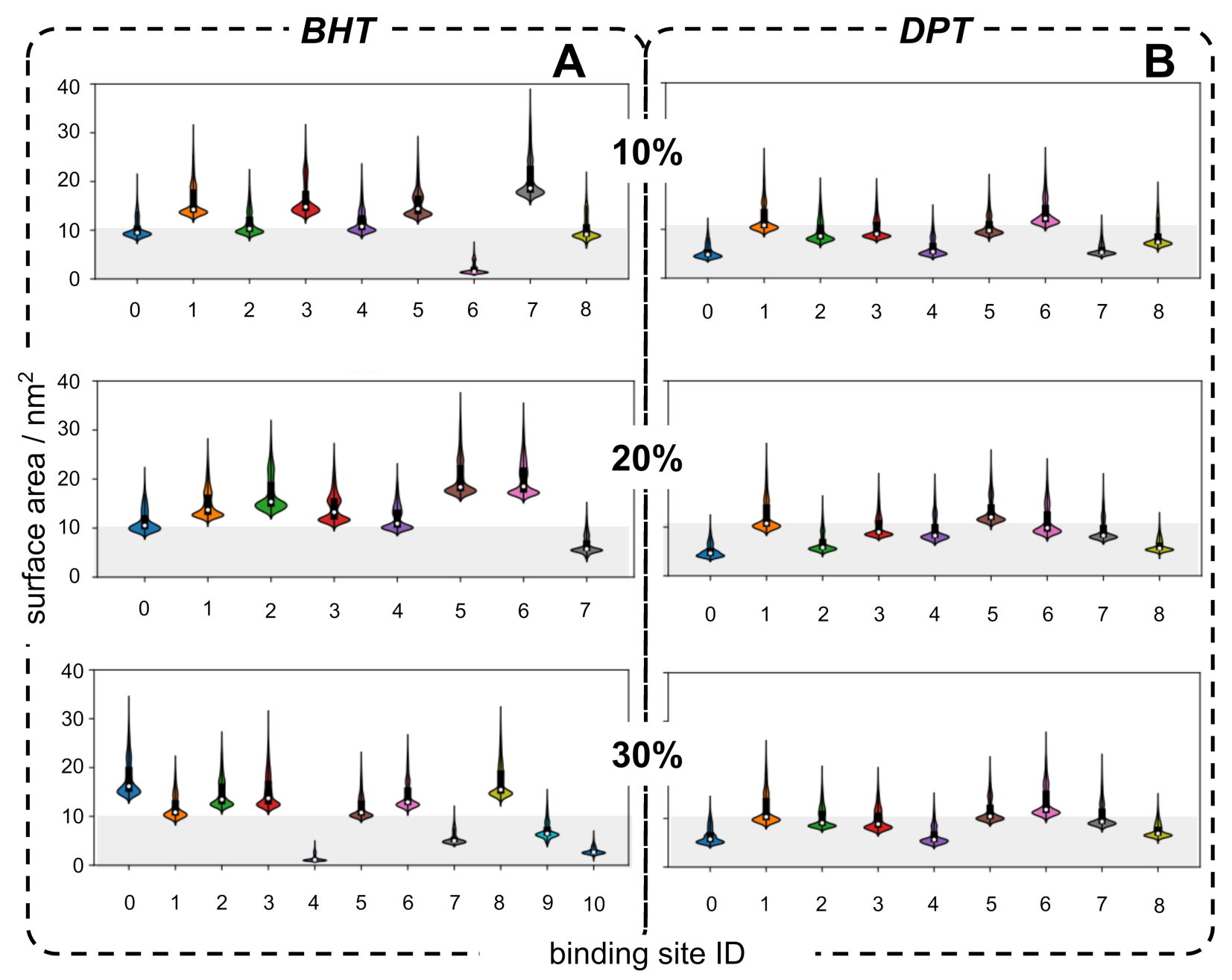

Figure S4: BHT has more large-area interaction sites with PR. A) Number of hopanoid binding sites for 10, 20, and $30 \mathrm{~mol} \%$ BHT in a monomeric PR system. B) Number of hopanoid binding sites for 10, 20, and $30 \mathrm{~mol} \% \mathrm{DPT}$ in a monomeric PR system. 


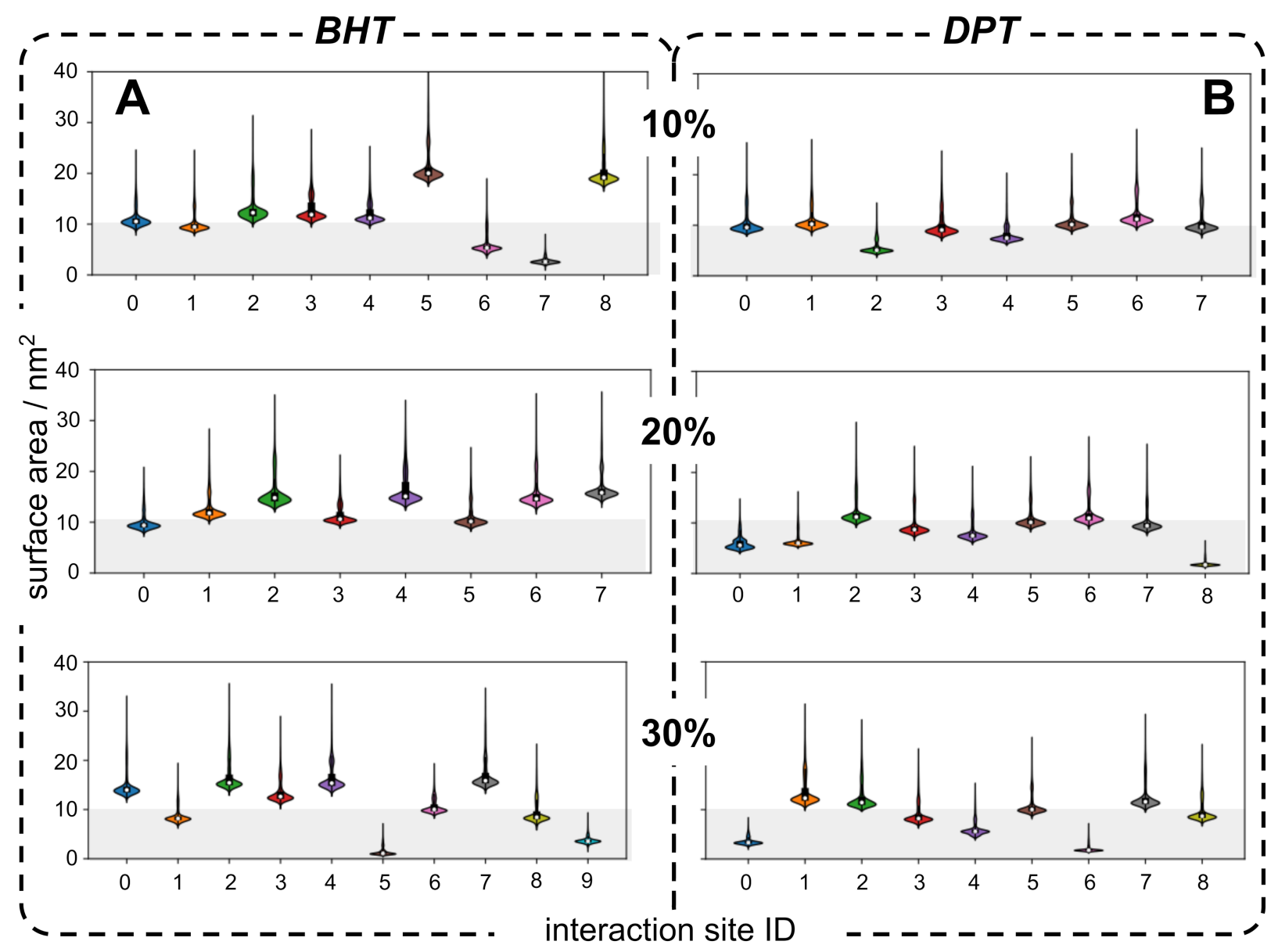

Figure S5: Dimers of PR have little effect on interaction sites of hopanoids. A) Binding surface area at 10 , 20 and $30 \mathrm{~mol} \%$ BHT for dimeric systems of PR. B) Binding surface area at 10, 20 and 30 mol\% DPT for dimeric systems of PR. 
A $\quad$ B
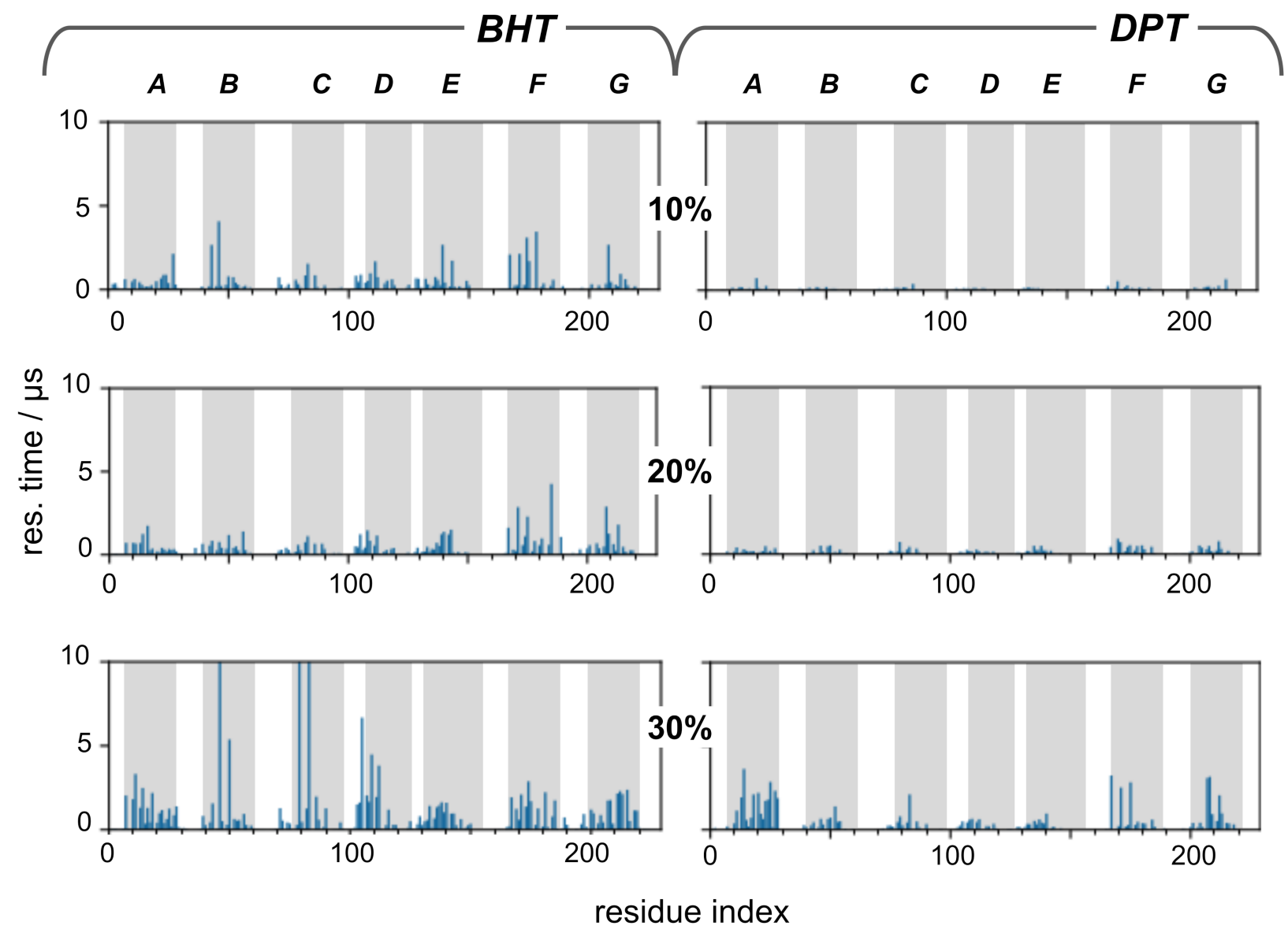

Figure S6: Dimers of PR shift locations of hopanoid interaction sites. A) Residence times for 10,20 and 30 mol\% BHT for dimeric systems of PR. B) Residence times for 10, 20 and 30 mol\% DPT for dimeric systems of PR. 
Table S1: Kinetics data for BHT interactions with PR monomer as a function of BHT concentration.

\begin{tabular}{|c|c|c|c|c|c|c|c|c|c|c|}
\hline \multirow{5}{*}{$10 \%$} & Binding site & 0 & 1 & 2 & 3 & 4 & 5 & 6 & 7 & 8 \\
\hline & Residence times & 1.3 & 7 & 0.5 & 0.8 & 2.4 & 1.1 & 0 & 7 & 0.3 \\
\hline & \# of BHT & 1.6 & 2.1 & 1.3 & 1.9 & 1.5 & 1.6 & 1 & 2.2 & 1.1 \\
\hline & koff & 0.797 & 0.072 & 1.895 & 1.328 & 0.409 & 0.951 & 39.803 & 0.09 & 3.277 \\
\hline & $\mathrm{R} 2$ & 0.9747 & 0.9892 & 0.9944 & 0.9976 & 0.9557 & 0.9122 & 0.9918 & 0.9927 & 0.995 \\
\hline
\end{tabular}

\begin{tabular}{|l|l|r|r|r|r|r|r|r|r|}
\hline Binding site & 0 & 1 & 2 & 3 & 4 & 5 & 6 & 7 \\
\hline Residence times & 4 & 0.4 & 0.5 & 5.3 & 0.6 & 5.7 & 7 & 1.7 \\
\cline { 2 - 9 } & \# of BHT & 1.9 & 1.6 & 2.8 & 2.2 & 1.6 & 3.9 & 3.2 & 1.1 \\
\cline { 2 - 9 } & koff & 0.248 & 2.3 & 1.858 & 0.189 & 1.653 & 0.175 & 0.12 & 0.575 \\
\hline R2 & 0.9682 & 0.9968 & 0.9743 & 0.9956 & 0.9677 & 0.9952 & 0.8885 & 0.9766 \\
\hline
\end{tabular}

\begin{tabular}{|l|l|r|r|r|r|r|r|r|r|r|r|r|}
\hline \multirow{3}{*}{$30 \%$} & Binding site & 0 & 1 & 2 & 3 & 4 & 5 & 6 & 7 & 8 & 9 & 10 \\
\cline { 2 - 12 } & Residence times & 7 & 2.7 & 3.1 & 7 & 0 & 2.6 & 5.7 & 0.8 & 7 & 1.1 & 0 \\
\cline { 2 - 12 } & \# of BHT & 4.3 & 2.6 & 2.6 & 3.1 & 0.8 & 2.3 & 4.3 & 1.6 & 3.9 & 1.5 & 0.8 \\
\cline { 2 - 12 } & koff & 0.104 & 0.367 & 0.319 & 0.017 & 25.91 & 0.385 & 0.174 & 1.332 & 0.093 & 0.89 & 91.504 \\
\hline R2 & 0.9925 & 0.9902 & 0.9955 & 0.9887 & 0.9973 & 0.9924 & 0.9948 & 0.9993 & 0.991 & 0.9949 & 0.9983 \\
\hline
\end{tabular}

Table S2: Kinetics data for DPT interactions with PR monomer as a function of DPT concentration.

\begin{tabular}{|r|r|r|r|r|r|r|r|r|r|}
\hline \multirow{4}{*}{$10 \%$} & 0 & 1 & 2 & 3 & 4 & 5 & 6 & 7 & 8 \\
\cline { 2 - 10 } & 0 & 0.3 & 0.3 & 0.1 & 0.4 & 0.1 & 0.4 & 0 & 0.1 \\
\cline { 2 - 10 } & 27.927 & 3.942 & 2.978 & 16.726 & 2.839 & 7.055 & 2.461 & 60.287 & 9.642 \\
\cline { 2 - 10 } & 0.9994 & 0.9988 & 0.9997 & 0.9901 & 0.9907 & 0.9999 & 0.9985 & 0.9998 & 0.9979 \\
\hline
\end{tabular}

\begin{tabular}{|c|c|c|c|c|c|c|c|c|c|}
\hline \multirow{5}{*}{$20 \%$} & 0 & 1 & 2 & 3 & 4 & 5 & 6 & 7 & 8 \\
\hline & 0.1 & 0.6 & 0.6 & 0.2 & 0.5 & 0.5 & 1.7 & 0.4 & 0 \\
\hline & 1.1 & 2.4 & 1.8 & 2 & 2.1 & 2.8 & 2 & 1.6 & 1 \\
\hline & 6.723 & 1.751 & 1.544 & 4.518 & 1.878 & 2.117 & 0.578 & 2.579 & 26.877 \\
\hline & 0.9992 & 0.9981 & 0.9991 & 0.9996 & 0.9993 & 0.999 & 0.4991 & 0.9984 & 0.9994 \\
\hline
\end{tabular}

\begin{tabular}{|r|r|r|r|r|r|r|r|r|r|}
\hline 0 & 1 & 2 & 3 & 4 & 5 & 6 & 7 & 8 \\
\cline { 2 - 9 } $30 \%$ & 0.4 & 0.9 & 0.4 & 3.3 & 2.1 & 0.9 & 0.7 & 0.5 & 0.1 \\
\cline { 2 - 9 } & 1.4 & 3 & 2.8 & 3.2 & 2.2 & 3.8 & 3 & 2.1 & 1 \\
\cline { 2 - 9 } & 2.461 & 1.1665 & 2.701 & 0.302 & 0.472 & 1.162 & 1.439 & 2.125 & 16.085 \\
\hline 0.9757 & 0.9983 & 0.9998 & 0.9744 & 0.995 & 0.9848 & 0.9567 & 0.9997 & 0.9996 \\
\hline
\end{tabular}


Table S3: Kinetics data for BHT interactions with PR dimer as a function of BHT concentration.

\begin{tabular}{|c|c|c|c|c|c|c|c|c|c|c|}
\hline \multirow{5}{*}{$10 \%$} & Binding site & 0 & 1 & 2 & 3 & 4 & 5 & 6 & 7 & 8 \\
\hline & Residence times & 0.7 & 0.5 & 2.2 & 4.4 & 0.8 & 2.2 & 0.4 & 0 & 4.9 \\
\hline & $\#$ of BHT & 1.4 & 1.2 & 1.6 & 1.5 & 1.4 & 2.9 & 1.1 & 0.5 & 1.9 \\
\hline & koff & 1.355 & 1.921 & 0.447 & 0.228 & 1.322 & 0.457 & 2.608 & 44.67 & 0.202 \\
\hline & $\mathrm{R} 2$ & 0.9992 & 0.997 & 0.9929 & 0.9518 & 0.9951 & 0.9981 & 0.9983 & 0.9927 & 0.9979 \\
\hline
\end{tabular}

\begin{tabular}{|l|l|r|r|r|r|r|r|r|r|}
\hline Binding site & 0 & 1 & 2 & 3 & 4 & 5 & 6 & 7 \\
\cline { 2 - 9 } $20 \%$ & 2 & 0.8 & 1.4 & 0.7 & 4.8 & 1.9 & 1.2 & 3.7 \\
\cline { 2 - 9 } & \#esidence times & 1.8 & 1.6 & 3.1 & 1.6 & 2.6 & 2.9 & 2.6 & 2.6 \\
\hline & koff BHT & 0.513 & 1.305 & 0.697 & 1.375 & 0.211 & 0.53 & 0.808 & 0.271 \\
\hline R2 & 0.964 & 0.9981 & 0.998 & 0.9645 & 0.9947 & 0.9524 & 0.999 & 0.9525 \\
\hline
\end{tabular}

\begin{tabular}{|l|l|r|r|r|r|r|r|r|r|r|r|}
\hline \multirow{5}{*}{$30 \%$} & 0 & 1 & 2 & 3 & 4 & 5 & 6 & 7 & 8 & 9 \\
\cline { 2 - 11 } & Rinding site & 2.3 & 6.7 & 4.4 & 1.9 & 6.5 & 0 & 1.4 & 3.8 & 4.4 & 0 \\
\cline { 2 - 11 } & \# of BHT & 3.4 & 2.4 & 3.1 & 2.3 & 3.7 & 1 & 2 & 5 & 2.1 & 1 \\
\cline { 2 - 11 } & koff & 0.432 & 0.149 & 0.23 & 0.528 & 0.154 & 22.128 & 0.716 & 0.263 & 0.229 & 69.748 \\
\hline R2 & 0.997 & 0.8606 & 0.993 & 0.9973 & 0.9853 & 0.9961 & 0.997 & 0.9963 & 0.988 & 0.9966 \\
\hline
\end{tabular}

Table S4: Kinetics data for DPT interactions with PR dimer as a function of DPT concentration.

\begin{tabular}{|r|r|r|r|r|r|r|r|r|}
\hline \multirow{7}{*}{$10 \%$} & 0 & 1 & 2 & 3 & 4 & 5 & 6 & 7 \\
\cline { 2 - 8 } & 0.1 & 0.5 & 0 & 0.3 & 0.2 & 0.2 & 0.2 & 0.6 \\
\cline { 2 - 8 } & 1.4 & 1.6 & 1 & 1.6 & 2.1 .4 & 1.9 & 1.5 & 1.2 \\
\hline 0.951 & 2.177 & 136.78 & 3.808 & 4.173 & 5.61 & 6.022 & 6.439 \\
\hline
\end{tabular}

\begin{tabular}{|r|r|r|r|r|r|r|r|r|r|}
\hline \multirow{5}{*}{$20 \%$} & 0 & 1 & 2 & 3 & 4 & 5 & 6 & 7 & 8 \\
\cline { 2 - 9 } & 0.2 & 0.6 & 0.6 & 1 & 0.5 & 0.3 & 0.5 & 0.7 & 0.1 \\
\cline { 2 - 9 } & 1.1 & 1.8 & 2.6 & 2 & 2.1 & 2.8 & 2.3 & 1.8 & 1 \\
\cline { 2 - 9 } & 6.588 & 1.784 & 1.627 & 0.972 & 2.102 & 2.915 & 1.95 & 1.535 & 14.912 \\
\hline 0.999 & 0.9956 & 0.9991 & 0.9982 & 0.9998 & 0.9998 & 0.999 & 0.9983 & 0.9936 \\
\hline
\end{tabular}

\begin{tabular}{|r|r|r|r|r|r|r|r|r|r|}
\hline 0 & 1 & 2 & 3 & 4 & 5 & 6 & 7 & 8 \\
\hline 0 & 3.7 & 2.7 & 1.9 & 0.5 & 0.7 & 0.1 & 0.6 & 1.6 \\
\cline { 2 - 9 } $30 \%$ & 1 & 3.4 & 3.3 & 2.8 & 2 & 3.7 & 1 & 3 & 2.1 \\
\cline { 2 - 9 } & 168.23 & 0.271 & 0.377 & 0.53 & 1.835 & 1.507 & 14.788 & 1.645 & 0.634 \\
\hline & 0.999 & 0.9969 & 0.9856 & 0.9779 & 0.9986 & 0.9827 & 0.9961 & 0.608 & 0.9909 \\
\hline
\end{tabular}

